\title{
Exact Formulas and Simple CP dependence of Neutrino Oscillation Probabilities in Matter with Constant Density
}

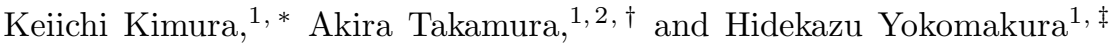 \\ ${ }^{1}$ Department of Physics, Nagoya University, Nagoya, 464-8602, Japan \\ ${ }^{2}$ Department of Mathematics, Toyota National Collage of Technology Eisei-cho 2-1, Toyota-shi, 471-8525, Japan
}

\begin{abstract}
We investigate neutrino oscillations in constant matter within the context of the standard three neutrino scenario. We derive an exact and simple formula for the oscillation probability applicable to all channels. In the standard parametrization, the probability for $\nu_{e} \rightarrow \nu_{\mu}$ transition can be written in the form $P\left(\nu_{e} \rightarrow \nu_{\mu}\right)=A_{e \mu} \cos \delta+B_{e \mu} \sin \delta+C_{e \mu}$ without any approximation using CP phase $\delta$. For $\nu_{\mu} \rightarrow \nu_{\tau}$ transition, the linear term of $\cos 2 \delta$ is added and the probability can be written in the form $P\left(\nu_{\mu} \rightarrow \nu_{\tau}\right)=A_{\mu \tau} \cos \delta+B_{\mu \tau} \sin \delta+C_{\mu \tau}+D_{\mu \tau} \cos 2 \delta$. We give the CP dependences of the probability for other channels. We show that the probability for each channel in matter has the same form with respect to $\delta$ as in vacuum. It means that matter effects just modify the coefficients $A, B, C$ and $D$. We also give the exact expression of the coefficients for each channel. Furthermore, we show that our results with respect to $\mathrm{CP}$ dependences are reproduced from the effective mixing angles and the effective CP phase calculated by Zaglauer and Schwarzer. Through the calculation, a new identity is obtained by dividing the Naumov-Harrison-Scott identity by the Toshev identity.
\end{abstract}

PACS numbers: $14.60 . \mathrm{Pq}$

\section{INTRODUCTION}

Strong evidences for neutrino oscillations have been accumulated and they indicate the finite masses and mixings of neutrinos. The first evidence is the observation of zenith-angle dependence of the atmospheric neutrino deficit [1], which is consistent with $\nu_{\mu} \rightarrow \nu_{\tau}$ transition with the mass difference and the mixing as

$$
\Delta_{32} \sim 3 \times 10^{-3} \mathrm{eV}^{2}, \quad \sin ^{2} 2 \theta_{23} \sim 1,
$$

where $\Delta_{i j}=m_{i}^{2}-m_{j}^{2}$ and $m_{i}$ is neutrino mass. The second evidence is the solar neutrino deficit [2], which is consistent with $\nu_{e} \rightarrow \nu_{\mu} / \nu_{\tau}$ transition. Recent reports for the measurements of CC and NC interactions in the SNO experiment 3] are consistent with the Standard Solar Model and strongly suggest the LMA MSW solution

$$
\Delta_{21} \sim 7 \times 10^{-5} \mathrm{eV}^{2}, \quad \sin ^{2} 2 \theta_{12} \sim 0.8
$$

to the solar neutrino problem. Thus, the information on neutrino masses and mixings (Maki-Nakagawa-Sakata matrix) [4 has been increased considerably. The main physics goals in future experiments are the determination of the unknown parameters $\theta_{13}$ (only upper bound $\sin ^{2} 2 \theta_{13}<0.1$ is obtained [5]) and the $\mathrm{CP}$ phase $\delta$ in addition to the high precision measurement of the parameters in (11) and (2). In particular, the observation of $\delta$ is quite interesting from the point of view that $\delta$ is related to the origin of the matter in the universe. In order to get at the CP phase $\delta$, a considerable number of studies have been made on $\mathrm{CP}$ violation [6, 8, 8, 9, 10.

\footnotetext{
*E-mail address: kimukei@eken.phys.nagova-u.ac.jp

${ }^{\dagger}$ E-mail address: takamura@eken.phvs.nagova-u.ac.jp

‡E-mail address: yoko@eken.phys.nagoya-u.ac.jp
}

Moreover several experiments using neutrino beam are planned 11, 12. In such experiments with high energy neutrino beam, matter effects cannot be neglected and prevent the precise measurement of pure CP effects. In this paper we derive an exact and simple formula of oscillation probabilities for all channels.

Before giving our results, let us give the present status of exact formulas associated with oscillation probability in constant matter. The mass eigenvalues have been given by Barger et al. [13]. Zaglauer and Schwarzer 14. have calculated effective mixing angles and effective $\mathrm{CP}$ phase in the standard parametrization. Furthermore, the elements of the MNS matrix have been calculated by Xing [15] in parametrization independent way. However, these exact formulas obtained by diagonalizing the Hamiltonian, are complicated and it is not easy to obtain useful information on matter effects and the $\mathrm{CP}$ phase. Ohlsson and Snellman [16] have directly calculated the amplitude without diagonalizing the Hamiltonian although the CP phase is not considered (which is similar to our result as shown in Sec.III).

Recently, by comparing the Hamiltonian in matter and that in vacuum, Naumov [17] and Harrison and Scott 18 have presented simple and elegant identity

$$
\tilde{\Delta}_{12} \tilde{\Delta}_{23} \tilde{\Delta}_{31} \tilde{J}=\Delta_{12} \Delta_{23} \Delta_{31} J
$$

where $J$ is the Jarlskog factor [19] and tilde represents the quantities in matter. We have investigated the matter enhancement of $\tilde{J}$ in Ref. 20] by using this identity. Furthermore, the enhancement of T-violating quantity $\Delta P_{T}=P\left(\nu_{e} \rightarrow \nu_{\mu}\right)-P\left(\nu_{\mu} \rightarrow \nu_{e}\right)$ has been also investigated by Parke and Weiler [21] and Akhmedov et al. 22 .

In our previous paper [23], we have found other identities on the product of the MNS matrix elements by comparing the Hamiltonian in matter and that in vacuum. 
From these identities, we have presented the exact formula for $P\left(\nu_{e} \rightarrow \nu_{\mu}\right)$ and we have definitely separated pure CP effects from matter effects. In the standard parametrization, we have also shown that the probability for $\nu_{e} \rightarrow \nu_{\mu}$ transition can be written in the form [33] 23.

$$
P\left(\nu_{e} \rightarrow \nu_{\mu}\right)=A_{e \mu} \cos \delta+B_{e \mu} \sin \delta+C_{e \mu} .
$$

It has also been found that CP trajectory proposed by Minakata and Nunokawa 24 is exactly elliptic in biprobability space when $\delta$ changes from 0 to $2 \pi$.

In this paper, extending our previous results [23], we derive an exact and simple formula of the oscillation probabilities for all channels. We also find that the probability for each channel in matter has the same form with respect to the $\mathrm{CP}$ phase $\delta$ as in vacuum. For example, the probability for $\nu_{\mu} \rightarrow \nu_{\tau}$ transition can be written as

$$
P\left(\nu_{\mu} \rightarrow \nu_{\tau}\right)=A_{\mu \tau} \cos \delta+B_{\mu \tau} \sin \delta+C_{\mu \tau}+D_{\mu \tau} \cos 2 \delta,
$$

both in vacuum and in matter. Namely, matter effects just modify $A_{\mu \tau}, B_{\mu \tau}, C_{\mu \tau}$ and $D_{\mu \tau}$. We also give the exact expression of these coefficients. Furthermore, we show that our results with respect to $\mathrm{CP}$ dependences are reproduced from the effective mixing angles and effective CP phase calculated by Zaglauer and Schwarzer [14]. Although the effective $\mathrm{CP}$ phase are complicated, the $\mathrm{CP}$ dependence of probability is simplified for all channels. This is guaranteed by the Toshev identity

$$
\tilde{s}_{23} \tilde{c}_{23} \sin \tilde{\delta}=\tilde{s}_{23} \tilde{c}_{23} \sin \tilde{\delta}
$$

Finally, we obtain a new identity

$$
\tilde{\Delta}_{12} \tilde{\Delta}_{23} \tilde{\Delta}_{31} \tilde{s}_{12} \tilde{c}_{12} \tilde{s}_{13} \tilde{c}_{13}^{2}=\Delta_{12} \Delta_{23} \Delta_{31} s_{12} c_{12} s_{13} c_{13}^{2},
$$

related to the 1-2 mixing and the 1-3 mixing by dividing the Naumov-Harrison-Scott identity by the Toshev identity.

\section{CP DEPENDENCES OF OSCILLATION PROBABILITIES IN VACUUM}

In this section, we review the $\mathrm{CP}$ dependences of the oscillation probabilities in vacuum. The Hamiltonian in vacuum

$$
H=\left(\begin{array}{ccc}
H_{e e} & H_{e \mu} & H_{e \tau} \\
H_{\mu e} & H_{\mu \mu} & H_{\mu \tau} \\
H_{\tau e} & H_{\tau \mu} & H_{\tau \tau}
\end{array}\right)
$$

is diagonalized as

$$
U^{\dagger} H U=\frac{1}{2 E}\left(\begin{array}{ccc}
0 & & \\
& \Delta_{21} & \\
& & \Delta_{31}
\end{array}\right)
$$

where $U$ is the MNS matrix which connects flavor eigenstates with mass eigenstates [34]. Then, the amplitude of $\nu_{\alpha}$ to $\nu_{\beta}$ transition after transporting the distance $L$ is

$$
A\left(\nu_{\alpha} \rightarrow \nu_{\beta}\right)=\sum_{i=1}^{3} U_{\alpha i}^{*} e^{-i \frac{\Delta_{i 1}}{2 E} L} U_{\beta i}
$$

where $\alpha$ and $\beta$ denote flavor indices $e, \mu$ or $\tau$. And the oscillation probability is given by

$$
\begin{aligned}
& P\left(\nu_{\alpha} \rightarrow \nu_{\beta}\right)=\left|A\left(\nu_{\alpha} \rightarrow \nu_{\beta}\right)\right|^{2} \\
& =\delta_{\alpha \beta}-4 \sum_{(i j)}^{\text {cyclic }} \operatorname{Re} J_{\alpha \beta}^{i j} \sin ^{2} \Delta_{i j}^{\prime} \pm 2 \sum_{(i j)}^{\text {cyclic }} J \sin 2 \Delta_{i j}^{\prime}
\end{aligned}
$$

where

$$
\begin{aligned}
J_{\alpha \beta}^{i j} & \equiv U_{\alpha i} U_{\beta i}^{*}\left(U_{\alpha j} U_{\beta j}^{*}\right)^{*}, \quad J \equiv \operatorname{Im} J_{e \mu}^{12}, \\
\Delta_{i j}^{\prime} & \equiv \frac{\Delta_{i j} L}{4 E} \equiv \frac{\left(m_{i}^{2}-m_{j}^{2}\right) L}{4 E}
\end{aligned}
$$

The \pm sign of the third term takes $-(+)$ in the case that $(\alpha, \beta)$ is given by (anti) cyclic permutation of $(e, \mu)$. The cyclic sum is over $(i j)=(12),(23),(31)$.

Next let us calculate $\operatorname{Re} J_{e \mu}^{i j}$ and $J$ to study the CP dependences of probabilities in the standard parametrization 25],

$$
U_{\alpha i}=\left(\begin{array}{ccc}
c_{12} c_{13} & s_{12} c_{13} & s_{13} e^{-i \delta} \\
-s_{12} c_{23}-c_{12} s_{23} s_{13} e^{i \delta} & c_{12} c_{23}-s_{12} s_{23} s_{13} e^{i \delta} & s_{23} c_{13} \\
s_{12} s_{23}-c_{12} c_{23} s_{13} e^{i \delta} & -c_{12} s_{23}-s_{12} c_{23} s_{13} e^{i \delta} & c_{23} c_{13}
\end{array}\right)
$$

where $s_{i j} \equiv \sin \theta_{i j}, c_{i j} \equiv \cos \theta_{i j}$.

$\operatorname{Re} J_{e \mu}^{i j}$ are given by

$$
\begin{aligned}
& \operatorname{Re} J_{e \mu}^{12}=-\left(c_{12}^{2}-s_{12}^{2}\right) J_{r} \cos \delta+s_{12}^{2} c_{12}^{2} c_{13}^{2}\left(s_{23}^{2} s_{13}^{2}-c_{23}^{2}\right) \\
& \operatorname{Re} J_{e \mu}^{23}=J_{r} \cos \delta-s_{12}^{2} s_{23}^{2} s_{13}^{2} c_{13}^{2} \\
& \operatorname{Re} J_{e \mu}^{31}=-J_{r} \cos \delta-c_{12}^{2} s_{23}^{2} s_{13}^{2} c_{13}^{2}
\end{aligned}
$$


and $\operatorname{Re} J_{e \tau}^{i j}$ are given by

$$
\begin{aligned}
& \operatorname{Re} J_{e \tau}^{12}=\left(c_{12}^{2}-s_{12}^{2}\right) J_{r} \cos \delta+s_{12}^{2} c_{12}^{2} c_{13}^{2}\left(c_{23}^{2} s_{13}^{2}-s_{23}^{2}\right), \\
& \operatorname{Re} J_{e \tau}^{23}=-J_{r} \cos \delta-s_{12}^{2} c_{23}^{2} s_{13}^{2} c_{13}^{2}, \\
& \operatorname{Re} J_{e \tau}^{31}=J_{r} \cos \delta-c_{12}^{2} c_{23}^{2} s_{13}^{2} c_{13}^{2},
\end{aligned}
$$

and $\operatorname{Re} J_{\mu \tau}^{i j}$ are given by

$$
\begin{aligned}
\operatorname{Re} J_{\mu \tau}^{12} & =s_{12}^{2} c_{12}^{2} s_{23}^{2} c_{23}^{2}\left(1+s_{13}^{2}+s_{13}^{4}\right)-\left(s_{12}^{2} c_{12}^{2}+s_{23}^{2} c_{23}^{2}\right) s_{13}^{2} \\
& -\left(c_{12}^{2}-s_{12}^{2}\right)\left(c_{23}^{2}-s_{23}^{2}\right) s_{12} c_{12} s_{23} c_{23} s_{13}\left(1+s_{13}^{2}\right) \cos \delta+2 s_{12}^{2} c_{12}^{2} s_{23}^{2} c_{23}^{2} s_{13}^{2} \cos 2 \delta, \\
\operatorname{Re} J_{\mu \tau}^{23} & =-\left(c_{23}^{2}-s_{23}^{2}\right) J_{r} \cos \delta+s_{23}^{2} c_{23}^{2} c_{13}^{2}\left(s_{12}^{2} s_{13}^{2}-c_{12}^{2}\right), \\
\operatorname{Re} J_{\mu \tau}^{31} & =\left(c_{23}^{2}-s_{23}^{2}\right) J_{r} \cos \delta+s_{23}^{2} c_{23}^{2} c_{13}^{2}\left(c_{12}^{2} s_{13}^{2}-s_{12}^{2}\right),
\end{aligned}
$$

where $J_{r} \equiv s_{12} c_{12} s_{23} c_{23} s_{13} c_{13}^{2}$. We also obtain the Jarlskog factor,

$$
J=J_{r} \sin \delta .
$$

It should be noted that the replacements $s_{23} \rightarrow c_{23}$, $c_{23} \rightarrow-s_{23}$ in $\operatorname{Re} J_{e \mu}^{i j}$ leads to $\operatorname{Re} J_{e \tau}^{i j}$.

In summary, the $\mathrm{CP}$ dependences of transition probabilities are obtained by substituting (15)-(24) into (11) as

$$
\begin{aligned}
& P\left(\nu_{e} \rightarrow \nu_{\mu}\right)=A_{e \mu} \cos \delta+B \sin \delta+C_{e \mu} \\
& P\left(\nu_{e} \rightarrow \nu_{\tau}\right)=-A_{e \mu} \cos \delta-B \sin \delta+C_{e \tau} \\
& P\left(\nu_{\mu} \rightarrow \nu_{\tau}\right)=A_{\mu \tau} \cos \delta+B \sin \delta+C_{\mu \tau}+D \cos 2 \delta .
\end{aligned}
$$

$P\left(\nu_{e} \rightarrow \nu_{\mu}\right)$ and $P\left(\nu_{e} \rightarrow \nu_{\tau}\right)$ have only linear terms of $\cos \delta$ and $\sin \delta$. On the other hand, $P\left(\nu_{\mu} \rightarrow \nu_{\tau}\right)$ has also a term proportional to $\cos 2 \delta$ in addition to the terms of $\cos \delta$ and $\sin \delta$.

Next, let us consider survival probabilities. One can calculate the $\mathrm{CP}$ dependences from the unitarity and the transition probabilities. Those are given by

$$
\begin{aligned}
P\left(\nu_{e} \rightarrow \nu_{e}\right) & =C_{e e}, \\
P\left(\nu_{\mu} \rightarrow \nu_{\mu}\right) & =A_{\mu \mu} \cos \delta+C_{\mu \mu}-D \cos 2 \delta, \\
P\left(\nu_{\tau} \rightarrow \nu_{\tau}\right) & =A_{\tau \tau} \cos \delta+C_{\tau \tau}-D \cos 2 \delta,
\end{aligned}
$$

where the coefficients can be calculated from (15)-(24). Note that the term proportional to $\sin \delta$ disappears in survival probabilities. In particular, not only $\sin \delta$ term but also $\cos \delta$ term does not exist in $P\left(\nu_{e} \rightarrow \nu_{e}\right)$ [28]. In $P\left(\nu_{\mu} \rightarrow \nu_{\mu}\right)$ and $P\left(\nu_{\tau} \rightarrow \nu_{\tau}\right)$, the linear term of $\cos 2 \delta$ has opposite sign to that in $P\left(\nu_{\mu} \rightarrow \nu_{\tau}\right)$. The probabilities for $\mathrm{CP}$ conjugate and $\mathrm{T}$ conjugate channels are obtained from (25)-(30) by the exchange $\delta \rightarrow-\delta$.

\section{DERIVATION OF EXACT FORMULA FOR $P\left(\nu_{\alpha} \rightarrow \nu_{\beta}\right)$}

In this section, we present three identities on the product of the MNS matrix elements. Then, we derive an exact formula for the oscillation probability. Furthermore, we confirm that the Naumov-Harrison-Scott identity can be also derived from our identities.

The Hamiltonian in matter $\tilde{H}$ is given by

$$
\tilde{H}=H+\frac{1}{2 E}\left(\begin{array}{lll}
a & & \\
& 0 & \\
& & 0
\end{array}\right),
$$

where $H$ is the Hamiltonian in vacuum and is diagonalized as (9), $a \equiv 2 \sqrt{2} G_{F} N_{e} E$ is the matter potential, $G_{F}$ is the Fermi constant and $N_{e}$ is the electron density in matter. Note that the second term in (31) is energy independent because the matter potential $a$ is proportional to the energy $E$. The Hamiltonian in matter is diagonalized by the effective MNS matrix as

$$
\tilde{U}^{\dagger} \tilde{H} \tilde{U}=\frac{1}{2 E}\left(\begin{array}{ccc}
\lambda_{1} & & \\
& \lambda_{2} & \\
& & \lambda_{3}
\end{array}\right),
$$

where $\lambda_{i}$ is mass eigenvalue in matter. The amplitude and probability for $\nu_{\alpha} \rightarrow \nu_{\beta}$ transition in matter are obtained by the replacements $m_{i}^{2} \rightarrow \lambda_{i}$ and $U \rightarrow \tilde{U}$ in (10)-(13).

As one of the approaches to derive the probability, one can calculate single $\tilde{U}_{\alpha i}$ by directly diagonalizing $\tilde{H}$ and calculate $\tilde{J}_{\alpha \beta}^{i j}$, which is the product of four $\tilde{U}$ s. However, the expressions for the probabilities obtained in this approach are complicated and it is not easy to extract the information on matter effects and $\delta$. Here, we derive the probability using another approach. Note that $\tilde{U}_{\alpha i} \tilde{U}_{\beta i}^{*}$ appears in the amplitude in (10) and the probability in (11). So, we only have to calculate the product $\tilde{U}_{\alpha i} \tilde{U}_{\beta i}^{*}$ and the expression for single $\tilde{U}_{\alpha i}$ is not necessary. We use the relation (31) in order to calculate $\tilde{U}_{\alpha i} \tilde{U}_{\beta i}^{*}$. We introduce $\tilde{p}_{\alpha \beta}$ and $\tilde{q}_{\alpha \beta}$ as

$$
\begin{aligned}
& \tilde{p}_{\alpha \beta}=2 E \tilde{H}_{\alpha \beta}, \\
& \tilde{q}_{\alpha \beta}=(2 E)^{2} \tilde{\mathcal{H}}_{\alpha \beta}=(2 E)^{2}\left(\tilde{H}_{\gamma \beta} \tilde{H}_{\alpha \gamma}-\tilde{H}_{\alpha \beta} \tilde{H}_{\gamma \gamma}\right)
\end{aligned}
$$

where $(\alpha \beta \gamma)=(e \mu \tau),(\mu \tau e),(\tau e \mu)$. Then, we obtain three kinds of identities on $\tilde{U}_{\alpha i} \tilde{U}_{\beta i}^{*}$. First, from unitarity 
we obtain

$$
\tilde{U}_{\alpha 1} \tilde{U}_{\beta 1}^{*}+\tilde{U}_{\alpha 2} \tilde{U}_{\beta 2}^{*}+\tilde{U}_{\alpha 3} \tilde{U}_{\beta 3}^{*}=\delta_{\alpha \beta}
$$

Second, $2 E \tilde{H}_{\alpha \beta}=\tilde{p}_{\alpha \beta}$ in (33), which is also seen in Ref. [29], is rewritten as

$$
\lambda_{1} \tilde{U}_{\alpha 1} \tilde{U}_{\beta 1}^{*}+\lambda_{2} \tilde{U}_{\alpha 2} \tilde{U}_{\beta 2}^{*}+\lambda_{3} \tilde{U}_{\alpha 3} \tilde{U}_{\beta 3}^{*}=\tilde{p}_{\alpha \beta} .
$$

Third, $(2 E)^{2} \tilde{\mathcal{H}}_{\alpha \beta}=\tilde{q}_{\alpha \beta}$ in $(34)$, is rewritten as

$$
\lambda_{2} \lambda_{3} \tilde{U}_{\alpha 1} \tilde{U}_{\beta 1}^{*}+\lambda_{3} \lambda_{1} \tilde{U}_{\alpha 2} \tilde{U}_{\beta 2}^{*}+\lambda_{1} \lambda_{2} \tilde{U}_{\alpha 3} \tilde{U}_{\beta 3}^{*}=\tilde{q}_{\alpha \beta},
$$

where we use the relation

$$
\begin{aligned}
(2 E)^{2} \tilde{\mathcal{H}} & =(2 E)^{2} \tilde{H}^{-1}(\operatorname{det} \tilde{H}) \\
& =\tilde{U} \operatorname{diag}\left(\frac{1}{\lambda_{1}}, \frac{1}{\lambda_{2}}, \frac{1}{\lambda_{3}}\right) \tilde{U}^{\dagger} \times \lambda_{1} \lambda_{2} \lambda_{3} .
\end{aligned}
$$

Eqs. (35)-(37) on $\tilde{U}_{\alpha i} \tilde{U}_{\beta i}^{*}$ can be simply solved as

$$
\tilde{U}_{\alpha i} \tilde{U}_{\beta i}^{*}=\frac{\tilde{p}_{\alpha \beta} \lambda_{i}+\tilde{q}_{\alpha \beta}-\delta_{\alpha \beta} \lambda_{i}\left(\lambda_{j}+\lambda_{k}\right)}{\tilde{\Delta}_{j i} \tilde{\Delta}_{k i}},
$$

where $(i j k)$ takes $(123),(231),(312)$. Note that $\tilde{p}_{\alpha \beta}$ and $\tilde{q}_{\alpha \beta}$ become constants in the following cases,

$$
\begin{aligned}
& \tilde{p}_{\alpha \beta}=p_{\alpha \beta} \quad(\alpha \neq e \text { or } \beta \neq e), \\
& \tilde{q}_{\alpha \beta}=q_{\alpha \beta} \quad(\alpha=e \text { or } \beta=e)
\end{aligned}
$$

from (31). In other cases, $\tilde{H}_{e e}$ is included in $\tilde{p}_{\alpha \beta}$ and $\tilde{q}_{\alpha \beta}$, and they depend on $a$ explicitly. Similar expression for $\tilde{U}_{\alpha i} \tilde{U}_{\beta i}^{*}$ in (39) has been obtained by Ohlsson and Snellman in Ref. [16 and also by Harrison and Scott [30] although the method of derivation is different. We have given an exact and simple formula of $P\left(\nu_{e} \rightarrow \nu_{\mu}\right)$ for the first time in Ref. 23. Here, we give the general formula of oscillation probability $P\left(\nu_{\alpha} \rightarrow \nu_{\beta}\right)$ applicable to all channels by calculating $\operatorname{Re} \tilde{J}_{\alpha \beta}^{i j}$ and $\tilde{J}$ from $\tilde{J}_{\alpha \beta}^{i j}=$ $\tilde{U}_{\alpha i} \tilde{U}_{\beta i}^{*}\left(\tilde{U}_{\alpha j} \tilde{U}_{\beta j}^{*}\right)^{*}$. In the case of $\alpha \neq \beta$, the expression is given by

$P\left(\nu_{\alpha} \rightarrow \nu_{\beta}\right)=-4 \sum_{(i j)}^{\text {cyclic }} \operatorname{Re} \tilde{J}_{\alpha \beta}^{i j} \sin ^{2} \tilde{\Delta}_{i j}^{\prime} \pm 2 \sum_{(i j)}^{\text {cyclic }} \tilde{J} \sin 2 \tilde{\Delta}_{i j}^{\prime}$,

where

$$
\begin{gathered}
\operatorname{Re} \tilde{J}_{\alpha \beta}^{i j}=\frac{\left|\tilde{p}_{\alpha \beta}\right|^{2} \lambda_{i} \lambda_{j}+\left|\tilde{q}_{\alpha \beta}\right|^{2}+\operatorname{Re}\left(\tilde{p}_{\alpha \beta} \tilde{q}_{\alpha \beta}^{*}\right)\left(\lambda_{i}+\lambda_{j}\right)}{\tilde{\Delta}_{i j} \tilde{\Delta}_{12} \tilde{\Delta}_{23} \tilde{\Delta}_{31}} \\
\tilde{J}=\frac{\operatorname{Im}\left(\tilde{p}_{e \mu} \tilde{q}_{e \mu}^{*}\right)}{\tilde{\Delta}_{12} \tilde{\Delta}_{23} \tilde{\Delta}_{31}}, \quad \tilde{\Delta}_{i j}^{\prime} \equiv \frac{\tilde{\Delta}_{i j} L}{4 E}
\end{gathered}
$$

The concrete expression for $\lambda_{i}$ [35] is calculated by the MNS matrix elements and the masses in vacuum as

$$
\begin{aligned}
& \lambda_{1}=\frac{1}{3} s-\frac{1}{3} \sqrt{s^{2}-3 t}\left[u+\sqrt{3\left(1-u^{2}\right)}\right], \\
& \lambda_{2}=\frac{1}{3} s-\frac{1}{3} \sqrt{s^{2}-3 t}\left[u-\sqrt{3\left(1-u^{2}\right)}\right], \\
& \lambda_{3}=\frac{1}{3} s+\frac{2}{3} u \sqrt{s^{2}-3 t},
\end{aligned}
$$

where $s, t, u$ are given by

$$
\begin{aligned}
& s=\Delta_{21}+\Delta_{31}+a \\
& t=\Delta_{21} \Delta_{31}+a\left[\Delta_{21}\left(1-s_{12}^{2} c_{13}^{2}\right)+\Delta_{31}\left(1-s_{13}^{2}\right)\right] \\
& u=\cos \left[\frac{1}{3} \cos ^{-1}\left(\frac{2 s^{3}-9 s t+27 a \Delta_{21} \Delta_{31} c_{12}^{2} c_{13}^{2}}{2\left(s^{2}-3 t\right)^{3 / 2}}\right)\right]
\end{aligned}
$$

in the standard parametrization 13, 14, 15. One can see that the effective masses in matter do not depend on the CP phase $\delta$.

Finally, we reproduce the Naumov-Harrison-Scott identity from (44) as

$$
\begin{aligned}
\tilde{\Delta}_{12} \tilde{\Delta}_{23} \tilde{\Delta}_{31} \tilde{J} & =\frac{1}{(2 E)^{3}} \operatorname{Im}\left[\tilde{H}_{e \mu}\left(\tilde{H}_{e \tau} \tilde{H}_{\tau \mu}-\tilde{H}_{e \mu} \tilde{H}_{\tau \tau}\right)^{*}\right] \\
& =\frac{1}{(2 E)^{3}} \operatorname{Im}\left[\tilde{H}_{e \mu} \tilde{H}_{\mu \tau} \tilde{H}_{\tau e}\right] \\
& =\frac{1}{(2 E)^{3}} \operatorname{Im}\left[H_{e \mu} H_{\mu \tau} H_{\tau e}\right] \\
& =\Delta_{12} \Delta_{23} \Delta_{31} J
\end{aligned}
$$

Thus, the Naumov-Harrison-Scott identity is easily derived from the identities which we used.

\section{CP DEPENDENCES OF OSCILLATION PROBABILITIES IN MATTER}

In this section, we calculate the oscillation probabilities in all channels by using (42)-(44) and investigate their CP dependences.

$$
P\left(\nu_{e} \rightarrow \nu_{\mu}\right)
$$

Let us consider the $\mathrm{CP}$ dependence of $P\left(\nu_{e} \rightarrow \nu_{\mu}\right)$ which is the best channel for the observation of $\mathrm{CP}$ violation. First, we calculate $\tilde{p}_{e \mu}$ and $\tilde{q}_{e \mu}$ from (36) and (37). As $\tilde{p}_{e \mu}$ and $\tilde{q}_{e \mu}$ do not include $\tilde{H}_{e e}$, they are equivalent to the constants $p_{e \mu}$ and $q_{e \mu}$, which are represented by the quantities in vacuum. Therefore, we obtain

$$
\begin{aligned}
& p_{e \mu}=\Delta_{21} U_{e 2} U_{\mu 2}^{*}+\Delta_{31} U_{e 3} U_{\mu 3}^{*}, \\
& q_{e \mu}=\Delta_{31} \Delta_{21} U_{e 1} U_{\mu 1}^{*}
\end{aligned}
$$

from (36) and (37). Next, we divide $p_{e \mu}$ and $q_{e \mu}$ into the terms including and not including $\delta$ as

$$
p_{e \mu}=p_{e \mu}^{a} e^{-i \delta}+p_{e \mu}^{b}, \quad q_{e \mu}=q_{e \mu}^{a} e^{-i \delta}+q_{e \mu}^{b},
$$

where $p_{e \mu}^{a}, p_{e \mu}^{b}, q_{e \mu}^{a}$ and $q_{e \mu}^{b}$ are real numbers given by

$$
\begin{aligned}
& p_{e \mu}^{a}=\left(\Delta_{31}-\Delta_{21} s_{12}^{2}\right) s_{23} s_{13} c_{13}, \\
& p_{e \mu}^{b}=\Delta_{21} s_{12} c_{12} c_{23} c_{13}, \\
& q_{e \mu}^{a}=-\Delta_{31} \Delta_{21} c_{12}^{2} s_{23} s_{13} c_{13}, \\
& q_{e \mu}^{b}=-\Delta_{31} \Delta_{21} s_{12} c_{12} c_{23} c_{13},
\end{aligned}
$$


in the standard parametrization.

The oscillation probability can be written in the form

$$
P\left(\nu_{e} \rightarrow \nu_{\mu}\right)=\tilde{A}_{e \mu} \cos \delta+\tilde{B} \sin \delta+\tilde{C}_{e \mu}
$$

from (42)-(44). Note that $P\left(\nu_{e} \rightarrow \nu_{\mu}\right)$ is exactly linear in $\sin \delta$ and $\cos \delta$.

Next, we give the exact expression for $\tilde{A}_{e \mu}, \tilde{B}$ and $\tilde{C}_{e \mu}$. We rewrite them in the product of the oscillation part which depends on $L$ and $\left(\tilde{A}_{r}\right)_{i j}, \tilde{B}_{r}$ and $\left(\tilde{C}_{r}\right)_{i j}$ as

$$
\begin{aligned}
\tilde{A}_{e \mu} & =\sum_{(i j)}^{\text {cyclic }}\left(\tilde{A}_{r}\right)_{i j} \sin ^{2} \tilde{\Delta}_{i j}^{\prime}, \\
\tilde{B} & =\sum_{(i j)}^{\text {cyclic }} \tilde{B}_{r} \sin 2 \tilde{\Delta}_{i j}^{\prime}, \\
\tilde{C}_{e \mu} & =\sum_{(i j)}^{\text {cyclic }}\left(\tilde{C}_{r}\right)_{i j} \sin ^{2} \tilde{\Delta}_{i j}^{\prime} .
\end{aligned}
$$

$\tilde{B}$ is expressed in the form of the sum as 61). Under the condition $x+y+z=0$, the relation

$$
\sin 2 x+\sin 2 y+\sin 2 z=-4 \sin x \sin y \sin z
$$

holds and $\tilde{B}$ from (61) is rewritten in the form of product as

$$
\tilde{B}=\sum_{(i j)}^{\text {cyclic }} \tilde{B}_{r} \sin 2 \tilde{\Delta}_{i j}^{\prime}=-4 \tilde{B}_{r} \sin \tilde{\Delta}_{12}^{\prime} \sin \tilde{\Delta}_{23}^{\prime} \sin \tilde{\Delta}_{31}^{\prime}(64)
$$

$\tilde{A}_{e \mu}$ is rewritten in the same way. Under the same condition as in deriving $\tilde{B}$, the relation

$$
\sin ^{2} x=-(\sin x \sin y \cos z+\sin x \cos y \sin z)
$$

holds and $\tilde{A}_{e \mu}$ is rewritten as

$$
\begin{aligned}
& \tilde{A}_{e \mu}=\sum_{(i j)}^{\text {cyclic }}\left(\tilde{A}_{r}\right)_{i j} \sin ^{2} \tilde{\Delta}_{i j}^{\prime} \\
& =-\sum_{(i j k)}^{\text {cyclic }}\left[\left(\tilde{A}_{r}\right)_{j k}+\left(\tilde{A}_{r}\right)_{k i}\right] \cos \tilde{\Delta}_{i j}^{\prime} \sin \tilde{\Delta}_{j k}^{\prime} \sin \tilde{\Delta}_{k i}^{\prime}
\end{aligned}
$$

Substituting (55)-(58) into $p_{e \mu}$ and $q_{e \mu}$ in (42)-(44), $\tilde{A}_{e \mu}$, $\tilde{B}$ and $\tilde{C}_{e \mu}$ are rewritten with the masses and mixings as

$$
\begin{aligned}
\tilde{A}_{e \mu} & =\sum_{(i j k)}^{\text {cyclic }} \frac{-8\left[J_{r} \Delta_{21} \Delta_{31} \lambda_{k}\left(\lambda_{k}-\Delta_{31}\right)+\left(\tilde{A}_{e \mu}\right)_{k}\right]}{\tilde{\Delta}_{j k}^{2} \tilde{\Delta}_{k i}^{2}} \cos \tilde{\Delta}_{i j}^{\prime} \sin \tilde{\Delta}_{j k}^{\prime} \sin \tilde{\Delta}_{k i}^{\prime}, \\
\tilde{B} & =\frac{8 J_{r} \Delta_{12} \Delta_{23} \Delta_{31}}{\tilde{\Delta}_{12} \tilde{\Delta}_{23} \tilde{\Delta}_{31}} \sin \tilde{\Delta}_{12}^{\prime} \sin \tilde{\Delta}_{23}^{\prime} \sin \tilde{\Delta}_{31}^{\prime}, \\
\tilde{C}_{e \mu} & =\sum_{(i j)}^{\text {cyclic }} \frac{-4\left[\Delta_{31}^{2} s_{13}^{2} s_{23}^{2} c_{13}^{2} \lambda_{i} \lambda_{j}+\left(\tilde{C}_{e \mu}\right)_{i j}\right]}{\tilde{\Delta}_{i j} \tilde{\Delta}_{12} \tilde{\Delta}_{23} \tilde{\Delta}_{31}} \sin ^{2} \tilde{\Delta}_{i j}^{\prime} .
\end{aligned}
$$

See Appendix for the expression of $\left(\tilde{A}_{e \mu}\right)_{k}$ and $\left(\tilde{C}_{e \mu}\right)_{i j}$. Note that these expressions (67)-(69) are still exact. In the limit of small $\Delta_{21},\left(\tilde{A}_{e \mu}\right)_{k}$ and $\left(\tilde{C}_{e \mu}\right)_{i j}$ are higher order in $\Delta_{21}$ and can be ignored.

Finally, we obtain the well known approximate formula by neglecting the smallest effective mass. Considering the energy of neutrino $(E \geq 10 \mathrm{GeV})$ in neutrino factory experiment and the earth matter density $\left(\rho \simeq 2.8 \mathrm{~g} / \mathrm{cm}^{3}\right)$, matter potential is given by

$$
\begin{aligned}
a & =2 \sqrt{2} G_{F} N_{e} E=7.56 \times 10^{-5} \mathrm{eV}^{2} \frac{\rho}{\mathrm{gcm}^{-3}} \frac{E}{\mathrm{GeV}} \\
& \geq 2.1 \times 10^{-3} \mathrm{eV}^{2} .
\end{aligned}
$$

It means that the smallest effective mass $\lambda_{1}$ is almost equivalent to $\Delta_{21}$. Other effective masses $\lambda_{2}$ and $\lambda_{3}$, correspond to $a$ or $\Delta_{31}$. Accordingly, the coefficients are approximated by

$$
\begin{aligned}
\tilde{A}_{e \mu} & \simeq \frac{8 J_{r} \Delta_{21} \Delta_{31}}{a\left(\Delta_{31}-a\right)} \cos \Delta_{31}^{\prime} \sin a^{\prime} \sin \left(\Delta_{31}-a\right)^{\prime}, \\
\tilde{B} & \simeq \frac{8 J_{r} \Delta_{21} \Delta_{31}}{a\left(\Delta_{31}-a\right)} \sin \Delta_{31}^{\prime} \sin a^{\prime} \sin \left(\Delta_{31}-a\right)^{\prime}, \\
\tilde{C}_{e \mu} & \simeq \frac{4 \Delta_{31}^{2} s_{23}^{2} s_{13}^{2} c_{13}^{2}}{\left(\Delta_{31}-a\right)^{2}} \sin ^{2}\left(\Delta_{31}-a\right)^{\prime} .
\end{aligned}
$$

Although the approximate formula derived here is in agreement with the ones seen in Ref. [7, 8], the derivation is rather simple. The coefficients $\tilde{A}_{e \mu}$ and $\tilde{B}$ responsible for $\delta$ are $O\left(\Delta_{21} s_{13}\right)$. On the other hand, $\tilde{C}_{e \mu}$ is $O\left(s_{13}^{2}\right)$. Therefore, the terms related to $\delta$ become relatively large in the case of small $s_{13}$. 


$$
P\left(\nu_{e} \rightarrow \nu_{\tau}\right)
$$

In this subsection, we calculate $P\left(\nu_{e} \rightarrow \nu_{\tau}\right)$ in the same way as $P\left(\nu_{e} \rightarrow \nu_{\mu}\right)$. We need to learn $\tilde{U}_{e i} \tilde{U}_{\tau i}^{*}$ for this purpose.

At first, we consider $\tilde{p}_{e \tau}$ and $\tilde{q}_{e \tau}$. Since $\tilde{p}_{e \tau}$ and $\tilde{q}_{e \tau}$ do not include $\tilde{H}_{e e}$, they are equivalent to the constants $p_{e \tau}$ and $q_{e \tau}$. Therefore, we obtain

$$
\begin{aligned}
& p_{e \tau}=\Delta_{21} U_{e 2} U_{\tau 2}^{*}+\Delta_{31} U_{e 3} U_{\tau 3}^{*}, \\
& q_{e \tau}=\Delta_{31} \Delta_{21} U_{e 1} U_{\tau 1}^{*}
\end{aligned}
$$

from (36) and (37). Next, we divide $p_{e \tau}$ and $q_{e \tau}$ into the terms including and not including $\delta$ as

$$
p_{e \tau}=p_{e \tau}^{a} e^{-i \delta}+p_{e \tau}^{b}, \quad q_{e \tau}=q_{e \tau}^{a} e^{-i \delta}+q_{e \tau}^{b},
$$

where $p_{e \tau}^{a}, p_{e \tau}^{b}, q_{e \tau}^{a}$ and $q_{e \tau}^{b}$ are real numbers given by

$$
\begin{aligned}
& p_{e \tau}^{a}=\left(\Delta_{31}-\Delta_{21} s_{12}^{2}\right) c_{23} s_{13} c_{13}, \\
& p_{e \tau}^{b}=-\Delta_{21} s_{12} c_{12} s_{23} c_{13} \\
& q_{e \tau}^{a}=-\Delta_{31} \Delta_{21} c_{12}^{2} c_{23} s_{13} c_{13}, \\
& q_{e \tau}^{b}=\Delta_{31} \Delta_{21} s_{12} c_{12} s_{23} c_{13},
\end{aligned}
$$

in the standard parametrization. Note that $p_{e \tau}$ and $q_{e \tau}$ are obtained by the replacements $s_{23} \rightarrow c_{23}, c_{23} \rightarrow-s_{23}$ in $p_{e \mu}$ and $q_{e \mu}$. The coefficients of $\cos \delta$ and $\sin \delta$ have the opposite sign by the replacements, from (67) and 687). Therefore, $P\left(\nu_{e} \rightarrow \nu_{\tau}\right)$ can be also written in the form

$$
P\left(\nu_{e} \rightarrow \nu_{\tau}\right)=-\tilde{A}_{e \mu} \cos \delta-\tilde{B} \sin \delta+\tilde{C}_{e \tau},
$$

where

$$
\tilde{C}_{e \tau}=\sum_{(i j)}^{\text {cyclic }} \frac{-4\left[\Delta_{31}^{2} s_{13}^{2} c_{23}^{2} c_{13}^{2} \lambda_{i} \lambda_{j}+\left(\tilde{C}_{e \tau}\right)_{i j}\right]}{\tilde{\Delta}_{i j} \tilde{\Delta}_{12} \tilde{\Delta}_{23} \tilde{\Delta}_{31}} \sin ^{2} \tilde{\Delta}_{i j}^{\prime}
$$

and the concrete expression for $\left(\tilde{C}_{e \tau}\right)_{i j}$ is given in Appendix. As for $\tilde{A}_{e \mu}$ and $\tilde{B}$, see $(67)$ and $(68)$. Note that this expression is also exact. In the limit of small $\Delta_{21}$, $\left(\tilde{C}_{e \tau}\right)_{i j}$ is high order in $\Delta_{21}$ and can be neglected.
As in the case of $P\left(\nu_{e} \rightarrow \nu_{\mu}\right)$, we obtain the approximate formula as

$$
\tilde{C}_{e \tau} \simeq \frac{4 \Delta_{31}^{2} c_{23}^{2} s_{13}^{2} c_{13}^{2}}{\left(\Delta_{31}-a\right)^{2}} \sin ^{2}\left(\Delta_{31}-a\right)^{\prime},
$$

by neglecting the smallest effective mass $\lambda_{1} \simeq \Delta_{21}$. As the result, the leading term $\tilde{C}_{e \tau}$ which is not related to $\delta$ is $O\left(s_{13}^{2}\right)$ as in the case of $P\left(\nu_{e} \rightarrow \nu_{\mu}\right)$. It means that $\mathrm{CP}$ effect becomes relatively large also in this channel.

$$
P\left(\nu_{\mu} \rightarrow \nu_{\tau}\right)
$$

In this subsection, we calculate $P\left(\nu_{\mu} \rightarrow \nu_{\tau}\right)$. The expression for the probability of this channel is useful to analyze the appearance experiments of MINOS [31] and CNGS [32]. It is also needed for the analysis of neutrino factory experiments in which $\nu_{\tau}$ is produced by the high energy $\nu_{\mu}$ beam. In order to derive $P\left(\nu_{\mu} \rightarrow \nu_{\tau}\right)$, we calculate $\tilde{U}_{\mu i} \tilde{U}_{\tau i}^{*}$. Let us first consider $\tilde{p}_{\mu \tau}$ and $\tilde{q}_{\mu \tau}$ in $\tilde{U}_{\mu i} \tilde{U}_{\tau i}^{*}$. Since $\tilde{q}_{\mu \tau}$ includes $H_{e e}$, it is not constant and depends on the matter potential $a$ explicitly as

$$
\begin{aligned}
\frac{1}{(2 E)^{2}} \tilde{q}_{\mu \tau} & =\tilde{\mathcal{H}}_{\mu \tau}=\left\{H_{e \tau} H_{\mu e}-\left(H_{e e}+\frac{a}{2 E}\right) H_{\mu \tau}\right\} \\
& =\frac{1}{(2 E)^{2}}\left(q_{\mu \tau}-a p_{\mu \tau}\right) .
\end{aligned}
$$

Then, $\tilde{U}_{\mu i} \tilde{U}_{\tau i}^{*}$ is given by

$$
\tilde{U}_{\mu i} \tilde{U}_{\tau i}^{*}=\frac{\tilde{p}_{\mu \tau} \lambda_{i}+\tilde{q}_{\mu \tau}}{\tilde{\Delta}_{j i} \tilde{\Delta}_{k i}}=\frac{p_{\mu \tau}\left(\lambda_{i}-a\right)+q_{\mu \tau}}{\tilde{\Delta}_{j i} \tilde{\Delta}_{k i}} .
$$

Here, the different point between $P\left(\nu_{e} \rightarrow \nu_{\mu}\right)$ and $P\left(\nu_{e} \rightarrow \nu_{\tau}\right)$ is that the coefficient of $p_{\mu \tau}$ is not $\lambda_{i}$ but $\lambda_{i}-a$. Fortunately, the matter potential $a$ does not depend on $\delta$, so we have only to investigate $p_{\mu \tau}$ and $q_{\mu \tau}$, in order to learn the CP dependence of the probability. In the standard parametrization, we obtain

$$
p_{\mu \tau}=p_{\mu \tau}^{a} e^{-i \delta}+p_{\mu \tau}^{b}+p_{\mu \tau}^{c} e^{i \delta}, \quad q_{\mu \tau}=q_{\mu \tau}^{a} e^{-i \delta}+q_{\mu \tau}^{b}+q_{\mu \tau}^{c} e^{i \delta},
$$

where

$$
\begin{aligned}
& p_{\mu \tau}^{a}=-\Delta_{21} s_{12} c_{12} c_{23}^{2} s_{13}, \quad p_{\mu \tau}^{b}=\left[\Delta_{31} c_{13}^{2}-\Delta_{21}\left(c_{12}^{2}-s_{12}^{2} s_{13}^{2}\right)\right] s_{23} c_{23}, \quad p_{\mu \tau}^{c}=\Delta_{21} s_{12} c_{12} s_{23}^{2} s_{13}, \\
& q_{\mu \tau}^{a}=\Delta_{31} \Delta_{21} s_{12} c_{12} c_{23}^{2} s_{13}, \quad q_{\mu \tau}^{b}=\Delta_{31} \Delta_{21}\left(-s_{12}^{2}+c_{12}^{2} s_{13}^{2}\right) s_{23} c_{23}, \quad q_{\mu \tau}^{c}=-\Delta_{31} \Delta_{21} s_{12} c_{12} s_{23}^{2} s_{13} .
\end{aligned}
$$

And then, the transition probability can be written in the form

$$
P_{\mu \tau}=\tilde{A}_{\mu \tau} \cos \delta+\tilde{B} \sin \delta+\tilde{C}_{\mu \tau}+\tilde{D} \cos 2 \delta,
$$


where we obtain the expression for the coefficients after some straightforward calculations as

$$
\begin{aligned}
\tilde{A}_{\mu \tau} & =\sum_{(i j k)}^{\text {cyclic }} \frac{-8\left[J_{r} \Delta_{21} \Delta_{31}\left(\lambda_{k}-a\right)\left(\lambda_{k}-a-\Delta_{31}\right)\left(c_{23}^{2}-s_{23}^{2}\right)+\left(\tilde{A}_{\mu \tau}\right)_{k}\right]}{\tilde{\Delta}_{j k}^{2} \tilde{\Delta}_{k i}^{2}} \cos \tilde{\Delta}_{i j}^{\prime} \sin \tilde{\Delta}_{j k}^{\prime} \sin \tilde{\Delta}_{k i}^{\prime}, \\
\tilde{C}_{\mu \tau} & =\sum_{(i j)}^{\text {cyclic }} \frac{-4\left[\Delta_{31}^{2} s_{23}^{2} c_{23}^{2} c_{13}^{4}\left(\lambda_{i}-a\right)\left(\lambda_{j}-a\right)+\left(\tilde{C}_{\mu \tau}\right)_{i j}\right]}{\tilde{\Delta}_{i j} \tilde{\Delta}_{12} \tilde{\Delta}_{23} \tilde{\Delta}_{31}} \sin ^{2} \tilde{\Delta}_{i j}^{\prime}, \\
\tilde{D} & =\sum_{(i j k)}^{\text {cyclic }} \frac{-8 \Delta_{21}^{2}\left(\lambda_{k}-a-\Delta_{31}\right)^{2} s_{12}^{2} c_{12}^{2} s_{23}^{2} c_{23}^{2} s_{13}^{2}}{\tilde{\Delta}_{j k}^{2} \tilde{\Delta}_{k i}^{2}} \cos \tilde{\Delta}_{i j}^{\prime} \sin \tilde{\Delta}_{j k}^{\prime} \sin \tilde{\Delta}_{k i}^{\prime},
\end{aligned}
$$

and the concrete expressions for $\left(\tilde{A}_{\mu \tau}\right)_{k}$ and $\left(\tilde{C}_{\mu \tau}\right)_{i j}$ are given in Appendix. As for $\tilde{B}$, see (68). Note that these expressions (90)-(92) are also exact. In the limit of small $\Delta_{21},\left(\tilde{A}_{\mu \tau}\right)_{k}$ and $\left(\widetilde{C}_{e \tau}\right)_{i j}$ are high order in $\Delta_{21}$ and can be neglected.

Here, let us comment on the magnitude of each term. $\tilde{D}$ is $O\left(\Delta_{21}^{2} s_{13}^{2}\right)$ and hence it is difficult to observe the term proportional to $\cos 2 \delta$. $\tilde{A}_{\mu \tau}$ is suppressed by $\Delta_{21} s_{13}$ as in the previous cases. In addition, $\tilde{A}_{\mu \tau}$ is also proportional to $c_{23}^{2}-s_{23}^{2}$. Since the $2-3$ mixing is almost maximal from the atmospheric neutrino experiments, $\tilde{A}_{\mu \tau}$ is considered to be rather small. On the other hand, $\tilde{C}_{\mu \tau}$ has not any suppression factor. Accordingly, the leading contribution comes from $\tilde{C}_{\mu \tau}$ and the next to leading comes from $\tilde{B}$. Other contributions from $\tilde{A}_{\mu \tau}$ and $\tilde{D}$ are extremely small and we give the approximate formula for $\tilde{C}_{\mu \tau}$.

As in the previous cases, we obtain

$$
\tilde{C}_{\mu \tau} \simeq 4 s_{23}^{2} c_{23}^{2} c_{13}^{4} \sin ^{2} \Delta_{31}^{\prime}
$$

by neglecting the smallest effective mass $\lambda_{1} \simeq \Delta_{21}$. We conclude that it is considerably difficult to observe the $\mathrm{CP}$ effects in the standard scenario.

$$
P\left(\nu_{e} \rightarrow \nu_{e}\right), P\left(\nu_{\mu} \rightarrow \nu_{\mu}\right) \text { and } P\left(\nu_{\tau} \rightarrow \nu_{\tau}\right)
$$

In this subsection, we calculate survival probabilities using unitarity and the transition probabilities derived in the previous subsections.

Let us first consider $P\left(\nu_{e} \rightarrow \nu_{e}\right)$. The expression for the probability of this channel is needed, for example in neutrino factory experiments with the high energy $\nu_{e}\left(\bar{\nu}_{e}\right)$ beam. In the standard scenario, the unitarity relation

$$
P\left(\nu_{e} \rightarrow \nu_{e}\right)=1-P\left(\nu_{e} \rightarrow \nu_{\mu}\right)-P\left(\nu_{e} \rightarrow \nu_{\tau}\right)
$$

holds. From (67) and (68) the coefficients of $\cos \delta$ and $\sin \delta$ are proportional to $J_{r}$ which includes $s_{23} c_{23}$. As $P\left(\nu_{e} \rightarrow \nu_{\tau}\right)$ is obtained from $P\left(\nu_{e} \rightarrow \nu_{\mu}\right)$ by the exchange $s_{23} \rightarrow c_{23}, c_{23} \rightarrow-s_{23}$ (i.e. $J_{r} \rightarrow-J_{r}$ ), one can understand that the terms proportional to $\cos \delta$ and $\sin \delta$ disappear in (94). Therefore, $P\left(\nu_{e} \rightarrow \nu_{e}\right)$ is completely independent of $\delta$ and can be written in the form

$$
P\left(\nu_{e} \rightarrow \nu_{e}\right)=\tilde{C}_{e e},
$$

as first pointed out in Ref. 28. Here,

$$
\begin{aligned}
& \tilde{C}_{e e}=1-\tilde{C}_{e \mu}-\tilde{C}_{e \tau} \\
& =1+\sum_{(i j)}^{\text {cyclic }} \frac{4\left[\Delta_{31}^{2} s_{13}^{2} c_{13}^{2} \lambda_{i} \lambda_{j}+\left(\tilde{C}_{e e}\right)_{i j}\right]}{\tilde{\Delta}_{i j} \tilde{\Delta}_{12} \tilde{\Delta}_{23} \tilde{\Delta}_{31}} \sin ^{2} \tilde{\Delta}_{i j}^{\prime}
\end{aligned}
$$

As for the concrete expression of $\left(\tilde{C}_{e e}\right)_{i j}$, see Appendix. Note that this expression is exact. In the limit of small $\Delta_{21},\left(\tilde{C}_{e e}\right)_{i j}$ are higher order in $\Delta_{21}$ and can be ignored. Furthermore, we obtain an approximate formula

$$
\tilde{C}_{e e} \simeq 1-\frac{4 \Delta_{31}^{2} s_{13}^{2} c_{13}^{2}}{\left(\Delta_{31}-a\right)^{2}} \sin ^{2}\left(\Delta_{31}-a\right)^{\prime},
$$

by neglecting the smallest effective mass $\lambda_{1} \simeq \Delta_{21}$. $P\left(\nu_{e} \rightarrow \nu_{e}\right)$ mainly depends on $s_{13}$. This channel may be interesting because $s_{13}$ is determined regardless of the ambiguity of $\delta$.

Next, we calculate $P\left(\nu_{\mu} \rightarrow \nu_{\mu}\right)$. From unitarity, the relation $P\left(\nu_{\mu} \rightarrow \nu_{\mu}\right)=1-P\left(\nu_{\mu} \rightarrow \nu_{e}\right)-P\left(\nu_{\mu} \rightarrow \nu_{\tau}\right)$ holds. Then, the term proportional to $\sin \delta$ disappears and the probability can be written in the form

$$
P\left(\nu_{\mu} \rightarrow \nu_{\mu}\right)=\tilde{A}_{\mu \mu} \cos \delta+\tilde{C}_{\mu \mu}-\tilde{D} \cos 2 \delta
$$

where the coefficients are given by

$$
\begin{aligned}
& \tilde{A}_{\mu \mu}=-\tilde{A}_{e \mu}-\tilde{A}_{\mu \tau} \simeq-\tilde{A}_{e \mu} \\
& \tilde{C}_{\mu \mu}=1-\tilde{C}_{e \mu}-\tilde{C}_{\mu \tau} \simeq 1-\tilde{C}_{\mu \tau} .
\end{aligned}
$$

In the approximation $(100)$ we use the fact that $\tilde{A}_{\mu \tau}$ is proportional to $c_{23}^{2}-s_{23}^{2}$ from $(90)$ and is very small in the case of maximal $2-3$ mixing. In the approximation (101), we use the fact that $\tilde{C}_{e \mu}$ is suppressed by $s_{13}^{2}$. As for $\tilde{D}$, it is equivalent to that in $P\left(\nu_{\mu} \rightarrow \nu_{\tau}\right)$.

Finally, it may be useful to study the CP dependence of $P\left(\nu_{\tau} \rightarrow \nu_{\tau}\right)$ although this channel is very difficult to observe. From unitarity, the relation $P\left(\nu_{\tau} \rightarrow \nu_{\tau}\right)=$ 
$1-P\left(\nu_{e} \rightarrow \nu_{\tau}\right)-P\left(\nu_{\mu} \rightarrow \nu_{\tau}\right)$ also holds. Then, the probability can be written in the form

$$
P\left(\nu_{\tau} \rightarrow \nu_{\tau}\right)=\tilde{A}_{\tau \tau} \cos \delta+\tilde{C}_{\tau \tau}-\tilde{D} \cos 2 \delta .
$$

The coefficients are given by

$$
\begin{aligned}
& \tilde{A}_{\tau \tau}=-\tilde{A}_{e \tau}-\tilde{A}_{\mu \tau} \simeq-\tilde{A}_{e \tau}, \\
& \tilde{C}_{\tau \tau}=1-\tilde{C}_{e \tau}-\tilde{C}_{\mu \tau} \simeq 1-\tilde{C}_{\mu \tau} .
\end{aligned}
$$

Here, $\tilde{D}$ is also the same as that in $P\left(\nu_{\mu} \rightarrow \nu_{\tau}\right)$.

\section{Summary of CP dependences in Matter}

Here, we summarize the CP dependences of the oscillation probabilities for all channels in matter. They are given by

$$
\begin{aligned}
& P\left(\nu_{e} \rightarrow \nu_{\mu}\right)=\tilde{A}_{e \mu} \cos \delta+\tilde{B} \sin \delta+\tilde{C}_{e \mu} \\
& P\left(\nu_{e} \rightarrow \nu_{\tau}\right)=-\tilde{A}_{e \mu} \cos \delta-\tilde{B} \sin \delta+\tilde{C}_{e \tau} \\
& P\left(\nu_{\mu} \rightarrow \nu_{\tau}\right)=\tilde{A}_{\mu \tau} \cos \delta+\tilde{B} \sin \delta+\tilde{C}_{\mu \tau}+\tilde{D} \cos 2 \delta \\
& P\left(\nu_{e} \rightarrow \nu_{e}\right)=\tilde{C}_{e e}
\end{aligned}
$$

$$
\begin{aligned}
& P\left(\nu_{\mu} \rightarrow \nu_{\mu}\right)=\tilde{A}_{\mu \mu} \cos \delta+\tilde{C}_{\mu \mu}-\tilde{D} \cos 2 \delta \\
& P\left(\nu_{\tau} \rightarrow \nu_{\tau}\right)=\tilde{A}_{\tau \tau} \cos \delta+\tilde{C}_{\tau \tau}-\tilde{D} \cos 2 \delta
\end{aligned}
$$

Comparing these results with (25)-(30), we find that the probability for each channel in matter has the same form with respect to the $\mathrm{CP}$ phase $\delta$ as in vacuum. It means that the matter effects just modify the coefficients. Finally, we comment that the probabilities for $\mathrm{T}$ conjugate channels are obtained from (105)-(110) by the replacement $\delta \rightarrow-\delta$ and those for $\overline{\mathrm{CP}}$ conjugate channels are obtained by $\delta \rightarrow-\delta$ and $a \rightarrow-a$.

\section{EFFECTIVE MIXING ANGLES AND EFFECTIVE CP PHASE}

In this section, we show that our results (105)-(110) with respect to the $\mathrm{CP}$ dependences are reproduced from the effective mixing angles and the effective $\mathrm{CP}$ phase calculated by Zaglauer and Schwarzer [14]. They have derived the effective mixing angles and $\mathrm{CP}$ phase from $\left|\tilde{U}_{\alpha \beta}\right|^{2}$ as

$$
\begin{aligned}
\sin ^{2} \tilde{\theta}_{13} & =\frac{\lambda_{3}^{2}-\alpha \lambda_{3}+\beta}{\tilde{\Delta}_{13} \tilde{\Delta}_{23}}, \\
\sin ^{2} \tilde{\theta}_{12} & =\frac{-\left(\lambda_{2}^{2}-\alpha \lambda_{2}+\beta\right) \tilde{\Delta}_{31}}{\left(\lambda_{1}^{2}-\alpha \lambda_{1}+\beta\right) \tilde{\Delta}_{32}-\left(\lambda_{2}^{2}-\alpha \lambda_{2}+\beta\right) \tilde{\Delta}_{31}}, \\
\sin ^{2} \tilde{\theta}_{23} & =\frac{G^{2} s_{23}^{2}+F^{2} c_{23}^{2}+2 G F s_{23} c_{23} \cos \delta}{G^{2}+F^{2}}, \\
e^{-i \tilde{\delta}} & =\frac{\left(G^{2} e^{-i \delta}-F^{2} e^{i \delta}\right) s_{23} c_{23}+G F\left(c_{23}^{2}-s_{23}^{2}\right)}{\sqrt{\left(G^{2} s_{23}^{2}+F^{2} c_{23}^{2}+2 G F s_{23} c_{23} \cos \delta\right)\left(G^{2} c_{23}^{2}+F^{2} s_{23}^{2}-2 G F s_{23} c_{23} \cos \delta\right)}},
\end{aligned}
$$

where $\alpha, \beta, G$ and $F$ are given by

$$
\begin{aligned}
\alpha & =p_{\mu \mu}+p_{\tau \tau}, \\
\beta & =q_{e e}, \\
G s_{23} & =p_{e \mu}^{a} \lambda_{3}+q_{e \mu}^{a}, \\
F c_{23} & =p_{e \mu}^{b} \lambda_{3}+q_{e \mu}^{b} .
\end{aligned}
$$

Let us here describe the derivation of the effective mixing angles [36]. We can easily calculate the 1-3 mixing from $\left|\tilde{U}_{e 3}\right|^{2}=\tilde{s}_{13}^{2}$. We can also calculate the 1-2 mixing by eliminating $\tilde{c}_{13}$ from $\left|\tilde{U}_{e 1}\right|^{2}=\tilde{c}_{12}^{2} \tilde{c}_{13}^{2}$ and $\left|\tilde{U}_{e 2}\right|^{2}=\tilde{s}_{12}^{2} \tilde{c}_{13}^{2}$. Furthermore, the 2-3 mixing can be calculated by eliminating $\tilde{c}_{13}$ from the relations,

$$
\left|\tilde{U}_{\mu 3}\right|^{2}=\frac{\left|\tilde{U}_{e 3} \tilde{U}_{\mu 3}^{*}\right|^{2}}{\left|\tilde{U}_{e 3}\right|^{2}}=\frac{\left|p_{e \mu} \lambda_{3}+q_{e \mu}\right|^{2}}{\tilde{\Delta}_{31} \tilde{\Delta}_{32}\left(\lambda_{3}^{2}-\alpha \lambda_{3}+\beta\right)}=\tilde{s}_{23}^{2} \tilde{c}_{13}^{2},
$$

$$
\left|\tilde{U}_{\tau 3}\right|^{2}=\frac{\left|\tilde{U}_{e 3} \tilde{U}_{\tau 3}^{*}\right|^{2}}{\left|\tilde{U}_{e 3}\right|^{2}}=\frac{\left|p_{e \tau} \lambda_{3}+q_{e \tau}\right|^{2}}{\tilde{\Delta}_{31} \tilde{\Delta}_{32}\left(\lambda_{3}^{2}-\alpha \lambda_{3}+\beta\right)}=\tilde{c}_{23}^{2} \tilde{c}_{13}^{2} .
$$

Next, we consider the $\mathrm{CP}$ dependence. One may think that the CP dependence of (114) is very complicated due to $\cos \delta$ in the denominator at a glance. However, our results (105)-(110) are very simple and the probabilities are linear in $\sin \delta, \cos \delta$ and $\cos 2 \delta$. It suggests that the complicated CP dependence in $e^{-i \tilde{\delta}}$ cancels with that in other terms. From (111)-(114),$\delta$ is only included in $\sin \tilde{\theta}_{23}$ except for $e^{-i \tilde{\delta}}$. Furthermore, the numerator of $\sin \tilde{\theta}_{23}$ is partially in agreement with the denominator of $e^{-i \tilde{\delta}}$. The product of $\tilde{s}_{23}, \tilde{c}_{23}$ and $e^{-i \tilde{\delta}}$ is calculated as

$$
\tilde{s}_{23} \tilde{c}_{23} e^{-i \tilde{\delta}}=\frac{\left(G^{2} e^{-i \delta}-F^{2} e^{i \delta}\right) s_{23} c_{23}+G F\left(c_{23}^{2}-s_{23}^{2}\right)}{G^{2}+F^{2}} .
$$


Note that the CP phase in the denominator of $e^{-i \tilde{\delta}}$ completely disappears. The imaginary part of $(121)$ is known as the Toshev identity 27]

$$
\tilde{s}_{23} \tilde{c}_{23} \sin \tilde{\delta}=\tilde{s}_{23} \tilde{c}_{23} \sin \tilde{\delta},
$$

which is independent of matter effects.

Finally, we demonstrate how the $\mathrm{CP}$ dependences of the oscillation probabilities become simple taking the channel $P\left(\nu_{e} \rightarrow \nu_{\mu}\right)$ as an example. In general, the oscillation probabilities in matter are also obtained from those in vacuum by the exchange $\theta_{i j} \rightarrow \tilde{\theta}_{i j}$ and $\delta \rightarrow \tilde{\delta}$. Namely, from 15)-(17) and (24), $P\left(\nu_{e} \rightarrow \nu_{\mu}\right)$ in matter is constituted by

$$
\begin{aligned}
\operatorname{Re} \tilde{J}_{e \mu}^{12}= & -\left(\tilde{c}_{12}^{2}-\tilde{s}_{12}^{2}\right) \tilde{J}_{r} \cos \tilde{\delta} \\
& +\tilde{s}_{12}^{2} \tilde{c}_{12}^{2} \tilde{c}_{13}^{2}\left(\tilde{s}_{23}^{2} \tilde{s}_{13}^{2}-\tilde{c}_{23}^{2}\right), \\
\operatorname{Re} \tilde{J}_{e \mu}^{23}= & \tilde{J}_{r} \cos \tilde{\delta}-\tilde{s}_{12}^{2} \tilde{s}_{23}^{2} \tilde{s}_{13}^{2} \tilde{c}_{13}^{2}, \\
\operatorname{Re} \tilde{J}_{e \mu}^{31}= & -\tilde{J}_{r} \cos \tilde{\delta}-\tilde{c}_{12}^{2} \tilde{s}_{23}^{2} \tilde{s}_{13}^{2} \tilde{c}_{13}^{2}, \\
\tilde{J}= & \tilde{J}_{r} \sin \tilde{\delta}
\end{aligned}
$$

We can see that $\sin \tilde{\delta}(\cos \tilde{\delta})$ always appears together with $\tilde{J}_{r}$ in $\operatorname{Re} \tilde{J}_{e \mu}^{i j}$ or in $\tilde{J}$. On the other hand, the CP dependence for the product $\tilde{s}_{23} \tilde{c}_{23} e^{-i \tilde{\delta}}$ become simple as shown in (121). Therefore, the CP dependence of the probability is simplified although that of the effective CP phase is complicated. Then, we obtain the same results

$$
P\left(\nu_{e} \rightarrow \nu_{\mu}\right)=\tilde{A}_{e \mu} \cos \delta+\tilde{B} \sin \delta+\tilde{C}_{e \mu}
$$

as (59). As for other channels, we obtain the same results as those calculated in our approach. This is guaranteed by the Toshev identity.

\section{NEW MATTER INVARIANT IDENTITY}

In the previous section, we rederived two matter invariant identities. One is the Naumov-Harisson-Scott iden- tity (51) and second is the Toshev identity (122). We obtain a new identity

$$
\tilde{\Delta}_{12} \tilde{\Delta}_{23} \tilde{\Delta}_{31} \tilde{s}_{12} \tilde{c}_{12} \tilde{s}_{13} \tilde{c}_{13}^{2}=\Delta_{12} \Delta_{23} \Delta_{31} s_{12} c_{12} s_{13} c_{13}^{2}
$$

related to the 1-2 mixing and the 1-3 mixing by dividing the Naumov-Harrison-Scott identity by the Toshev identity.

Here, we give another proof of this new identity (128). We can independently derive the identity (128) without using other identities in this proof. At first, taking $k_{1}, k_{2}$ as the eigenvalues of sub-matrix

$$
\frac{1}{2 E}\left(\begin{array}{cc}
H_{\mu \mu} & H_{\mu \tau} \\
H_{\tau \mu} & H_{\tau \tau}
\end{array}\right)
$$

we rewrite $\left|\tilde{U}_{e i}\right|^{2}$ obtained from (39) as

$$
\begin{aligned}
\left|\tilde{U}_{e i}\right|^{2} & =\frac{\lambda_{i}^{2}-\left(p_{\mu \mu}+p_{\tau \tau}\right) \lambda_{i}+q_{e e}}{\tilde{\Delta}_{j i} \tilde{\Delta}_{k j}} \\
& =\frac{\left(\lambda_{i}-k_{1}\right)\left(\lambda_{i}-k_{2}\right)}{\tilde{\Delta}_{j i} \tilde{\Delta}_{k i}} .
\end{aligned}
$$

Then, we obtain

$$
\begin{aligned}
& \left(\tilde{\Delta}_{12} \tilde{\Delta}_{23} \tilde{\Delta}_{31}\right)^{2}\left|\tilde{U}_{e 1}\right|^{2}\left|\tilde{U}_{e 2}\right|^{2}\left|\tilde{U}_{e 3}\right|^{2} \\
= & \prod_{i=1,2}\left(\lambda_{1}-k_{i}\right)\left(\lambda_{2}-k_{i}\right)\left(\lambda_{3}-k_{i}\right) \\
= & \prod_{i=1,2}\left(m_{1}^{2}-k_{i}\right)\left(m_{2}^{2}-k_{i}\right)\left(m_{3}^{2}-k_{i}\right)=\text { const. }
\end{aligned}
$$

where we use the relations obtained from the characteristic equation of Hamiltonian

$$
\begin{aligned}
\lambda_{1}+\lambda_{2}+\lambda_{3} & =m_{1}^{2}+m_{2}^{2}+m_{3}^{2}+a, \\
\lambda_{1} \lambda_{2}+\lambda_{2} \lambda_{3}+\lambda_{3} \lambda_{1} & =m_{1}^{2} m_{2}^{2}+m_{2}^{2} m_{3}^{2}+m_{3}^{2} m_{1}^{2}+a\left(k_{1}+k_{2}\right), \\
\lambda_{1} \lambda_{2} \lambda_{3} & =m_{1}^{2} m_{2}^{2} m_{3}^{2}+a k_{1} k_{2},
\end{aligned}
$$

from the first line to the second line in (131). Note that $k_{i}$ is the eigenvalue of the sub-matrix of Hamiltonian in vacuum and then does not depend on matter effects. Therefore, (131) is a matter invariant quantity and it is rewritten as (128).

\section{SUMMARY}

We summarize the results obtained in this paper. We consider the probabilities and the CP dependences for all channels within the context of standard three neutrino oscillations in constant matter. 
(i) We have derived an exact and simple formula for the oscillation probability applicable to all channels. We have also investigated the CP dependences of various probabilities using this formula. As the results, $P\left(\nu_{e} \rightarrow \nu_{\mu}\right)$ can be written in the form $A_{e \mu} \cos \delta+B \sin \delta+C_{e \mu} . P\left(\nu_{e} \rightarrow \nu_{\tau}\right)$ has the same form as $P\left(\nu_{e} \rightarrow \nu_{\mu}\right)$. On the other hand, the probability for $\nu_{\mu}$ to $\nu_{\tau}$ transition can be written as $P\left(\nu_{\mu} \rightarrow \nu_{\tau}\right)=A_{\mu \tau} \cos \delta+B \sin \delta+C_{\mu \tau}+D \cos 2 \delta$. In survival probabilities $P\left(\nu_{e} \rightarrow \nu_{e}\right), P\left(\nu_{\mu} \rightarrow \nu_{\mu}\right)$ and $P\left(\nu_{\tau} \rightarrow \nu_{\tau}\right)$, there is no $\sin \delta$ term. As for $P\left(\nu_{e} \rightarrow \nu_{e}\right), \cos \delta$ term also disappears and the probability is completely independent of $\delta$. We have found that the probability for each channel in matter has the same form with respect to the $\mathrm{CP}$ phase as in vacuum. That is, the matter effects just modify the coefficients $A, B, C$ and $D$. We have also given the exact expression for the coefficients in constant matter. (ii) We have shown that our results with respect to the $\mathrm{CP}$ dependences are reproduced from the effective mixing angles and CP phase derived by Zaglauer and Schwarzer. Although the effective CP phase are complicated, the CP dependence of probability is simplified for all channels. This is guaranteed by Toshev identity. Finally we have obtained a new identity related to the 1-2 mixing and the 1-3 mixing by dividing the Naumov-Harrison-Scott identity by the Toshev identity.

\section{Acknowledgments}

The authors would like to thank Prof. A. I. Sanda for making a number of helpful suggestions. We would like to thank Prof. H. Minakata and Prof. O. Yasuda for discussions and valuable comments.
[1] Super-Kamiokande Collaboration, Y. Fukuda et al., Phys. Rev. Lett. 81, 1562 (1998); Phys. Lett. B 433, 9 (1998); Phys. Lett. B 436, 33 (1998); Phys. Rev. Lett. 82, 2644 (1999).

[2] GALLEX Collaboration, W. Hampel et al., Phys. Lett. B 447, 127 (1999); SAGE Collaboration, J. N. Abdurashitov et al., Phys. Rev. C 60055801 (1999); Homestake Collaboration, B. T. Cleveland et al., Astrophys. J. 496, 505 (1998); Super-Kamiokande Collaboration, Y. Fukuda et al., Phys. Rev. Lett. 86, 5651 (2001); Super-Kamiokande Collaboration, S. Fukuda et al., hepex/0205075.

[3] SNO Collaboration, Q. R. Ahmad et al., Phys. Rev. Lett. 87, 071301 (2001); nucl-ex/0204008; nucl-ex/0204009.

[4] Z. Maki, M. Nakagawa and S. Sakata, Prog. Theor. Phys. 28, 870 (1962).

[5] CHOOZ Collaboration, M. Apollonio et al., Phys. Lett. B 420, 397 (1998); Phys. Lett. B 466, 415 (1999).

[6] For example

J. Arafune and J. Sato, Phys. Rev. D 55, 1653 (1997); H. Minakata and H. Nunokawa, Phys. Lett. B 413, 369 (1997); S. M. Bilenky, C. Giunti and W. Grimus, Phys. Rev. D 58, 033001 (1998); A. De Rujula, M. B. Gavela and P. Hernandez, Nucl. Phys. B 547, 21 (1999); K. Dick, M. Freund, M. Lindner and A. Romanino, Nucl. Phys. B 562, 29 (1999); M. Tanimoto, Phys. Lett. B 462, 115 (1999); A. Donini, M. B. Gavela, P. Hernandez and S. Rigolin, Nucl. Phys. B 574, 23 (2000); A. Romanino, Nucl. Phys. B 574, 675 (2000); O. Yasuda, Acta Phys. Polon. B 30, 3089 (1999); J. Sato, Nucl. Instrum. Meth. A 451, 36 (2000); M. Koike and J. Sato, Phys. Rev. D 61, 073012 (2000); Erratum ibid., D 62, 079903 (2000); H. Minakata and H. Nunokawa, Phys. Lett. B 495, 369 (2000); M. Koike, T. Ota and J. Sato, Phys. Rev. D 65, 053015 (2002); T. Miura, E. Takasugi, Y. Kuno and M. Yoshimura, Phys. Rev. D 64, 013002 (2001); I. Mocioiu and R. Shrock, JHEP 0111, 050 (2001); J. Pinney and O. Yasuda, Phys. Rev. D 64, 093008 (2001); P. Lipari, Phys. Rev. D 64, 033002 (2001); M. Freund, P. Huber and
M. Lindner, Nucl. Phys. B 615, 331 (2001); J. BurguetCastell et al., Nucl. Phys. B 608, 301 (2001); V. Barger, D. Marfatia and K. Whisnant, Phys. Rev. D 65, 073023 (2002); T. Kajita, H. Minakata and H. Nunokawa, Phys. Lett. B 528, 245 (2002).

[7] A. Cervera et al., Nucl. Phys. B 579, 17 (2000) [Erratumibid. B 593, 731 (2000)].

[8] M. Freund, Phys. Rev. D 64, 053003 (2001).

[9] J. Arafune, M. Koike and J. Sato, Phys. Rev. D 56, 3093 (1997); Erratum ibid., D 60, 119905 (1999).

[10] H. Minakata and H. Nunokawa, Phys. Rev. D 57, 4403 (1998).

[11] Y. Itow et al., hep-ex/0106019; M. Aoki et al., hepph/0104220; hep-ph/0112338.

[12] S. Geer, Phys. Rev. D 57, 6989 (1998).

[13] V. Barger, K. Whisnant, S. Pakvasa and R. J. N. Phillips, Phys. Rev. D 22, 2718 (1980).

[14] H. W. Zaglauer and K. H. Schwarzer, Z. Phys. C 40, 273 (1988).

[15] Z. Xing, Phys. Lett. B 487, 327 (2000).

[16] T. Ohlsson and H. Snellman, J. Math. Phys. 41, 2768 (2000); Phys. Lett. B 474, 153 (2000).

[17] V. A. Naumov, Int. J. Mod. Phys. D 1, 379 (1992).

[18] P. F. Harrison and W. G. Scott, Phys. Lett. B 476, 349 (2000).

[19] C. Jarlskog, Phys. Rev. Lett. 55, 1039 (1985).

[20] H. Yokomakura, K. Kimura and A. Takamura, Phys. Lett. B 496, 175 (2000).

[21] S. J. Parke and T. J. Weiler, Phys. Lett. B 501, 106 (2001).

[22] E. Kh. Akhmedov, P. Huber, M. Lindner and T. Ohlsson, Nucl. Phys. B 608, 394 (2001).

[23] K. Kimura, A. Takamura and H. Yokomakura, Phys. Lett. B 537, 86 (2002).

[24] H. Minakata and H. Nunokawa, JHEP 0110, 001 (2001); H. Minakata, H. Nunokawa and S. Parke, Phys. Lett. B 537, 249 (2002).

[25] Particle Data Group, D. E. Groom et al., Eur. Phys. J. C 15, 1 (2000). 
[26] P. I. Krastev and S. T. Petcov, Phys. Lett. B 205, 84 (1988).

[27] S. Toshev, Mod. Phys. Lett. A 6, 455 (1991).

[28] T. K. Kuo and J. Pantaleone, Phys. Rev. D 35, 3432 (1987).

[29] Z. Z. Xing, Phys. Rev. D 64, 033005 (2001).

[30] P. F. Harrison and W. G. Scott, Phys. Lett. B 535229 (2002).

[31] Numi MINOS project at Fermi National Accelerator Laboratory, http:/www-numi.fnal.gov/

[32] CERN Neutrinos to Gran Sasso, http://projcngs.web.cern.ch/proj-cngs/

[33] It has been first pointed out by Krastev and Petcov 26] that CP odd term is proportional to $\sin \delta$. It is easy to understand from the Naumov-Harrison-Scott identity [17, 18.

[34] Overall phase does not affect the probability from (11). By using this, the eigenvalues of the Hamiltonian are written with only mass differences subtracting $m_{1}^{2}$.

[35] When the calculation from amplitude to probability in matter, overall phase does not affect the probability. We remove the matter effects from $\mathrm{NC}$ interaction in (31) and $m_{1}^{2}$ in (32). Therefore, the expression for $\lambda_{i}$ does not depend on $m_{1}^{2}$ but mass differences.

[36] Zaglauer and Schwarzer derive effective mixing angles by using $\left|\tilde{U}_{\alpha \beta}\right|^{2}$. In this paper, the expression for $\tilde{U}_{\alpha i} \tilde{U}_{\beta i}^{*}$ is used to avoid the complexity of calculation.

\section{APPENDIX A: SUBLEADING TERMS IN THE $\tilde{A}_{\alpha \beta}, \tilde{C}_{\alpha \beta}$}

Here, we describe the terms which include higher order of $\Delta_{21}$ explicitly in $\tilde{A}_{\alpha \beta}$ and $\tilde{C}_{\alpha \beta}$.

First, the terms in $P\left(\nu_{e} \rightarrow \nu_{\mu}\right)$ are

$$
\begin{aligned}
\left(\tilde{A}_{e \mu}\right)_{k} & =\Delta_{21}^{2} J_{r} \times\left[\Delta_{31} \lambda_{k}\left(c_{12}^{2}-s_{12}^{2}\right)+\lambda_{k}^{2} s_{12}^{2}-\Delta_{31}^{2} c_{12}^{2}\right] \\
\left(\tilde{C}_{e \mu}\right)_{i j} & =\Delta_{21} s_{13}^{2} \times\left[\Delta_{31}\left\{-\lambda_{i}\left(\lambda_{j} s_{12}^{2}+\Delta_{31} c_{12}^{2}\right)-\lambda_{j}\left(\lambda_{i} s_{12}^{2}+\Delta_{31} c_{12}^{2}\right)\right\} s_{23}^{2} c_{13}^{2}\right] \\
& +\Delta_{21}^{2} \times\left[\left(\lambda_{i}-\Delta_{31}\right)\left(\lambda_{j}-\Delta_{31}\right) s_{12}^{2} c_{12}^{2} c_{23}^{2} c_{13}^{2}\right] \\
& +\Delta_{21}^{2} s_{13}^{2} \times\left[\left(\lambda_{i} s_{12}^{2}+\Delta_{31} c_{12}^{2}\right)\left(\lambda_{j} s_{12}^{2}+\Delta_{31} c_{12}^{2}\right) s_{23}^{2} c_{13}^{2}\right],
\end{aligned}
$$

where the second line of $\left(\tilde{C}_{e \mu}\right)_{i j}$ is relatively large in the case of $\Delta_{21} / \Delta_{31}<s_{13}$ so that there is no suppression factor $s_{13}^{2}$.

Second, the terms related to $P\left(\nu_{e} \rightarrow \nu_{\tau}\right)$ are

$$
\begin{aligned}
\left(\tilde{C}_{e \tau}\right)_{i j} & =\Delta_{21} s_{13}^{2} \times\left[\Delta_{31}\left\{-\lambda_{i}\left(\lambda_{j} s_{12}^{2}+\Delta_{31} c_{12}^{2}\right)-\lambda_{j}\left(\lambda_{i} s_{12}^{2}+\Delta_{31} c_{12}^{2}\right)\right\} c_{23}^{2} c_{13}^{2}\right] \\
& +\Delta_{21}^{2} \times\left[\left(\lambda_{i}-\Delta_{31}\right)\left(\lambda_{j}-\Delta_{31}\right) s_{12}^{2} c_{12}^{2} s_{23}^{2} c_{13}^{2}\right] \\
& +\Delta_{21}^{2} s_{13}^{2} \times\left[\left(\lambda_{i} s_{12}^{2}+\Delta_{31} c_{12}^{2}\right)\left(\lambda_{j} s_{12}^{2}+\Delta_{31} c_{12}^{2}\right) c_{23}^{2} c_{13}^{2}\right]
\end{aligned}
$$

where there is also no suppression factor $s_{13}^{2}$ in the second line.

Third, the terms related to $P\left(\nu_{\mu} \rightarrow \nu_{\tau}\right)$ are

$$
\begin{aligned}
\left(\tilde{A}_{\mu \tau}\right)_{k}= & -\Delta_{21}^{2} s_{13}\left(c_{23}^{2}-s_{23}^{2}\right) \\
& \times\left[s_{12} c_{12} s_{23} c_{23}\left(\lambda_{k}-a-\Delta_{31}\right)\left\{\Delta_{31}\left(s_{12}^{2}-s_{13}^{2} c_{12}^{2}\right)+\left(\lambda_{k}-a-\Delta_{31}\right) c_{12}^{2}\right\}\right] \\
\left(\tilde{C}_{\mu \tau}\right)_{i j}= & \Delta_{21} \times\left[\Delta_{31}\left\{\Delta_{31}\left(\lambda_{i}+\lambda_{j}-2 a\right)\left(c_{13}^{2}+c_{12}^{2}\right)-2\left(\lambda_{i}-a\right)\left(\lambda_{j}-a\right) c_{12}^{2}\right\} s_{23}^{2} c_{23}^{2} c_{13}^{2}\right] \\
+ & \Delta_{21} s_{13}^{2} \times\left[\Delta_{31}\left\{-\Delta_{31}\left(\lambda_{i}+\lambda_{j}-2 a\right)+2\left(\lambda_{i}-a\right)\left(\lambda_{j}-a\right)\right\} s_{12}^{2} s_{23}^{2} c_{23}^{2} c_{13}^{2}\right] \\
+ & \Delta_{21}^{2} \times\left[\left\{\Delta_{31} c_{13}^{2}+\left(\lambda_{i}-a-\Delta_{31}\right) c_{12}^{2}\right\}\left\{\Delta_{31} c_{13}^{2}+\left(\lambda_{j}-a-\Delta_{31}\right) c_{12}^{2}\right\} s_{23}^{2} c_{23}^{2}\right] \\
+ & \Delta_{21}^{2} s_{13}^{2} \times\left[-\Delta_{31}\left(\lambda_{i}+\lambda_{j}-2 a-2 \Delta_{31}\right) s_{12}^{2} s_{23}^{2} c_{23}^{2} c_{13}^{2}\right. \\
& \left.\quad+\left(\lambda_{i}-a-\Delta_{31}\right)\left(\lambda_{j}-a-\Delta_{31}\right)\left\{s_{12}^{2} c_{12}^{2}\left(c_{23}^{2}-s_{23}^{2}\right)+s_{12}^{4} s_{23}^{2} c_{23}^{2} s_{13}^{2}\right\}\right] .
\end{aligned}
$$

Finally, the terms related to $P\left(\nu_{e} \rightarrow \nu_{e}\right)$ are

$$
\begin{aligned}
\left(\tilde{C}_{e e}\right)_{i j} & =\Delta_{21} s_{13}^{2} \times\left[\Delta_{31}\left\{-\lambda_{i}\left(\lambda_{j} s_{12}^{2}+\Delta_{31} c_{12}^{2}\right)-\lambda_{j}\left(\lambda_{i} s_{12}^{2}+\Delta_{31} c_{12}^{2}\right)\right\} c_{13}^{2}\right] \\
& +\Delta_{21}^{2} \times\left[\left(\lambda_{i}-\Delta_{31}\right)\left(\lambda_{j}-\Delta_{31}\right) s_{12}^{2} c_{12}^{2} c_{13}^{2}\right] \\
& +\Delta_{21}^{2} s_{13}^{2} \times\left[\left(\lambda_{i} s_{12}^{2}+\Delta_{31} c_{12}^{2}\right)\left(\lambda_{j} s_{12}^{2}+\Delta_{31} c_{12}^{2}\right) c_{13}^{2}\right]
\end{aligned}
$$

where there is no suppression factor $s_{13}^{2}$ in the second 
contribution is relatively large in the case of $\Delta_{21} / \Delta_{31}<$ 